Int. J. Dev. Biol. 52: 893-901 (2008)

doi: $10.1387 / \mathrm{ijdb} .082683 \mathrm{mt}$

\title{
Retinoic acid metabolizing factor $x$ Cyp26c is specifically expressed in neuroectoderm and regulates anterior neural patterning in Xenopus laevis
}

\author{
MISAKI TANIBE ${ }^{1, \#,}$ TATSUO MICHIUE*,1,2,\#, AKIRA YUKITA ${ }^{1, \# \#, ~ H I R O K I ~ D A N N O 1 ~, ~ M A S A Y U K I ~ I K U Z A W A 2, ~}$ \\ SHOICHI ISHIURA ${ }^{1}$ and MAKOTO ASASHIMA*,1,2,3 \\ ${ }^{1}$ Graduate School of Arts and Sciences, The University of Tokyo, ${ }^{2}$ Organ Development Research Laboratory, National Institute of \\ Advanced Industrial Science and Technology (AIST) and ${ }^{3}$ Organ Regeneration project, International Cooperative Research Program \\ (ICORP), Japan Science and Technology Agency (JST), Japan
}

\begin{abstract}
Anterior-posterior neural patterning is determined during gastrulation when head structure is induced. Induction of anterior neural structures requires inhibition of Wnt signaling by several Wnt antagonists. We performed microarray analysis to isolate genes regulated by canonical Wnt signaling and abundantly expressed in the anterior neuroectoderm at the early neurula stage. We identified $x$ Cyp26c, a Cyp26 (RA-metabolizing protein)-family gene. In situ hybridization showed $x$ Cyp26c expression restricted to the anterior region of neurula, while $x C y p 26 a$ was expressed in both anterior and posterior regions. At the tadpole stage, $x C y p 26 c$ was also expressed in restricted sets of cranial nerves. Microarray, RT-PCR and in situ hybridization analyses revealed decreased $x C y p 26 c$ expression with overexpression of $\beta$-catenin, suggesting regulation by Wnt/ $\beta$-catenin signaling. We also assessed the effects of retinoic acid (RA) on $x$ Cyp26c expression. Embryos treated with $10^{-7} \mathrm{M}$ RA showed an anterior shift in the spatial expression of $x C y p 26 c$, reflecting a posteriorization effect. Conversely, expression patterns in embryos treated with more than $10^{-6} \mathrm{M}$ RA were less affected and remained restricted to the most anterior region. Moreover, injection of $x$ Cyp26c mRNA into animal poles caused head defects, and exogenous expression of $x$ Cyp26c rescued the posteriorizing effect of RA treatment. Taken together, these results implicated a role for $x C_{y p 26 c}$ in anterior patterning via RA signaling.
\end{abstract}

KEY WORDS: Xenopus, retinoic acid, Wnt signaling, AP neural patterning, rhombencephalon

\section{Introduction}

Anterior-posterior (AP) neural patterning and head structure formation is determined during gastrulation in Xenopus. Several intracellular signaling pathways are important in AP neural patterning including Wnt, fibroblast growth factor (FGF), and retinoic acid (RA) signaling (Niehrs, 1999; Sasai and DeRobertis, 1997). $\mathrm{RA}$ is a posteriorizing factor that induced loss of head due to posteriorization in Xenopus embryos treated with RA at the midblastula stage (Ruiz i Altaba and Jessell, 1990). RA can also increase types of organs induced by treatment with Activin, reflecting the posteriorization effect (Ariizumi and Asashima, 2001). Although not supported by direct evidence, a presumptive gradient of RA concentration along the midline is considered a determining factor for AP neural patterning. RA concentration is mainly determined by two major factors. The first is retinaldehyde dehydrogenase (RALDH). RA production largely occurs via the oxidative activity of RALDH. RaIDH2 is a RALDH-family gene expressed in a range of species including human, mouse, and rat (Zhao et al., 1996; Wang et al., 1996; Hsu et al., 2000). In Xenopus, RalDH2 is expressed around the yolk plug in the gastrula stage and overexpression of the gene caused an anterior shift in brain marker expression, implicating RaIDH2 function in AP neural patterning (Chen et al., 2001).

\footnotetext{
Abbreviations used in this paper: AP, anterior-posterior; FGF, fibroblast growth
} factor; RA, retinoic acid; RALDH, retinaldehyde dehydrogenase.

*Address correspondence to: Makoto Asashima and Tatsuo Michiue. 3-8-1 Komaba Meguro-ku, Tokyo 153-8902, Japan.

Tel: +81-3-5454-6632. Fax: +81-3-5454-4330. e-mail: asashi@bio.c.u-tokyo.ac.jp ; tmichiue@bio.c.u-tokyo.ac.jp

Notes: "These authors contributed equally to this paper. \#Present address: Department of Oral Histology, Matsumoto Dental University, Japan

Published online: 29 August 2008 
894 M. Tanibe et al.

hCYp26a MGLP-AL-LASALC-TFV--L-P--LLLFL-AAI-K--LWDLYCVSGRDRSCALPLPPGTMGFPFFGETLQMVLQRRKFLQMKRRKYGFIY MCYP26a MGLP-AL-LASALC-TFV--L-P--LLLFL-AAL-K- - LWDLYCVSSRDRSCALPLPPGTMGFPFFGETLQMVLQRRKFLQMKRRKYGFIY CYP26a MGLP-AL-LASALC-TFV--L-P--LLLFL-AAL-K--LWDLYCVSSRDRSCALPLP PGTMGFPFFGETLQMVLQRRKFLQMKRRKYGF IY $\begin{array}{ll}\text { CCYp26a } & \text { MG-FSAL-VASALC-TF---LLP--LLLFL-AAV-R--LWDLYCASGRDPSCPLPLP PGTMGLPFFGETLQMVLQRRKFLQMKRRKYGF IY } \\ \text { XCYP26a } & \text { MDLYT-L-LTSALC-TLA--L-P--LLLLLTAA--K--LWEVYCLRRKDAACANPLPPGTMGLPFFGETLOMVLORRRFLOVKRSOYGRIY }\end{array}$ ZCYP26a MGLYT-L-MVTFLC-TIV--L-PV-LL-FL-AAV-K--LWEMLMIRRVDPNCRSPLPPGTMGLPFIGETLQLILQRRKFLRMKRQKYGCIY $\begin{array}{ll}\text { hCyp26b } & \text { M-LFEGLDLVSAL-ATLAACLVSVTLLL----AVS-QQLWQLRWAATRDKSCKLPIPKGSMGFPLIGETGHWLLQGSGFQSSRREKYGNVF } \\ \text { mCyp26b } & \text { M-LFEGLELVSAL-ATLAACLVSVTLLL----AVS-QQLWQLRWAATRDKSCKLPIPKGSMGFPLIGETGHWLLQGSGFOSSRREKYGNVF }\end{array}$ ICYP2 6b M-LFEGLELVSAL-ATLAACLVSVTLLL- - -AVS-QQLWQLRWAATRDKSCKLPIPKGSMGFPLIGETGHWLLQGSGFOS SRREKYGNVF zCyp26b M-LFESFDLVSAL-ATLAACLVSMALLL---AVP-QQLWQLRWTATRDKSCKLPMPKGSMGFPI I GETCHWFFQGAGFHASRRQKYGNVF $\begin{array}{ll}\text { ZCYP26b } & \text { M-LFESFDLVSAL-ATLAACLVSMALLL----AVP-QQLWQLRWTATRDKSCKLPMPKGSMGFP I IGETCHWFFQGAGFHASRRQKYGNVF } \\ \text { BC111476 } & \text { MFLLEIS-YTSFFEATLTSVL-SLVLLL---AA-S-HQLWSLRWHSTRDRGSTLPLPKGSMGWPFFGETLHWLVQGSSFHSSRREKYGNVF }\end{array}$ hCyp 26 C MFPWGLSCL-SVLGAAGTALL-CAGLLL--SLA-- - QHLWTLRWMLSRDRASTLPLPKGSMGWPFFGETLHWLVQGSRFHSSRRERYGTVF ZCYp26C MFGHDFC-LVSALLSVADAVL-PTVLLL----AVSR-LLWEFRWSITRDKTCKLPLPQGSMGWPLVGETFHWLFQGSSFHISRREKHGNVF

hCyp26a KTHLFGRPTVRVMGADNVRRILLGEHRLVSVHWPASVRTILGSGCLSNLHDSSHKQRKKVIMRAFSREALECYVPVITEEVGSSLEQWLSC mCYp 26 a KTHLFGRPTVRVMGADNVRRI rCyp26a KTHI FGRPTVRVMGADNVRRIIIGEHRLVSVHWPASVRTILGAGCLSNLHDSSHKORKKVIMOAFNREALOCYVPVIAEEVSGCLEOWLSC CCYp26a KTIT Zсур 26 a hСур 26b mCyp 26b rСур 26b z Сур 26b BC11147 hCyp $26 c$

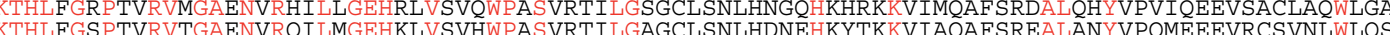
KTHLFGPTVRVTGAENVRQI LMGEHKLVSVHWPASVRTILGAGCLSNLHDNEHKYTKKVIAQAFSREALANYVPQMEEEVRCSVNLWLQS KTHLFGNPTVRVMGADNVRQI LLGEHKLVSVQWPASVRTILGSDTLSNVHGVQHKNKKKAIMRAFSRDALEHY I PVIQQEVKSAIQEWLQK
KTHLLGRPLIRVTGAENVRKI LMGEHHLVSTEWPRSTRMLLGPNTVSNSIGDIHRNKRKVFSKIFSHEALESYLPKIOLVIODTLRAW-SS KTHLLGRPLIRVTGAENVRKILLGEHQLVSTEWPRSARVLLGPNTVANS I GDIHRNKRKVFSKIFSHEALESYLPKIQLVIQDTLRAW-SS KTHLLGRPLIRVTGAENVRKILLGEHQLVSTEWPRSARVLLGPNTVANS I GDIHRNKRKVFSKIF SHEALESYLPKIQLVIQDTLRAW-SS KTHLIGRPLIRVTGAENVRKVLMGEHSLVTVDWPQSTSTLLGPNSLANS I GDIHRKRRKIFAKVFSHEALESYLPKIQQVIQETLRVW-SS KTHLLGKPVIRVTGAENIRKILLGEHSLVSTQWPQSTQMILGSNTLSNSIGELHRQKRKVMSKVLSSAALECYFPRIQEAVRWEVRGW-CR KTHLLGKPLIRVTGAENIRKILLGEHTVVCTQWPQSTRIILGPNTLVNSVGDLHKRKRKVLAKVFSRGALEAYLTRIQDVVKSEIAKW-CT

hCYp26a GERGLLVYPEVKRLMFRIAMRILLGCEPOLAGDGDSEOQLVEAFEEMTRNLFSLPIDVPFSGLYRGMKARNLIHARIEQNIRAKICGLRAS mсyp $26 a$ rСур 26 a cCyp26a XCYp26a XсYp26a hСyp 26b mCyp2 6b zCyp 26b ZCyp2 26 hCyp26c
Zcyp26c hCyp26a mсyp $26 a$ rCyp26a CCyp26a Zсур 26a hСур $26 \mathrm{~b}$ mCyp 26b rСyp 26b zCyp2 6b hCyp2 6 c

hСур 26 a

mсyp2 $6 a$

rCyp2 $6 a$

XCYp26a

Zcyp2 6 a

hCyp2 6b

mटyp 26b

zCyp2 $6 \mathrm{~b}$

BC11147 h⿻yp2 6 c Zcyp26c

hСур 26 a mсyp26a rटyp26a xCYp26a Zcyp26a hСур 26b mCyp 26b $\begin{array}{lll}\text { rCyp26b } & \text { RLDML-SLRYLDCVIKEVMRLFTPVSGGYRTVLQTFELDGFQI PKGWSVMYSIRDTHDTAPVFKDV-NVFDPDRFSQARSEDKDG--RFHY } \\ \text { zCyp26b } & \text { RLDSIISLKYLDCVIKEVLRLFAPVSGGYRIATOTFELDGVOVPKGWSVMYSIRDTHDTSAVFKDV-EAFDPDRFS PERSEDREG--RFHY }\end{array}$ BC111476 NLEKIKSLHYLECVVKEVLRLLPPVSGGYRTALQTFELDGYQIPKGWSVMYSIRDTHETAAVYQNA-EMFDPERF SSERDEGKLG- -KFN $\begin{array}{lll}\text { hCyp26C } & \text { SLAALGRLRYVDCVVKEVLRLLPPVSGGYRTALRTFELDGYOI PKGWSVMYS IRDTHETAAVYRSPPEGFDPERFGAARED SRGASSRFHY } \\ \text { ZCYP26C } & \text { SLEKLSOLSYLDCVVKEVLRFLPPVSGGYRTVLOTFELNGYOIPKGWSVMYSIRDTHETAEAYQNP-ELFDPDRFCVGREESK- -SERFSY }\end{array}$

hCYP26a IPFGGGLRSCVGKEFAKILTKIFTVETARHCDWOLLNGPPTMKTSPTVY - PVDNL - - - - - PARFTHF - - - - - - HGEI - - - - - - - - - - -

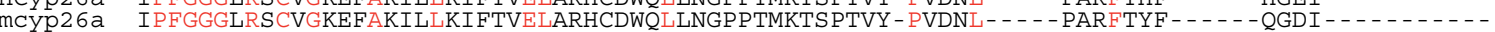
ICYP26a IPFGGGLRSCVGKEFAKILLKI FTVELARRCDWQLLNGPPTMKTSPPVY - PVDNL - - - PARFTHF-

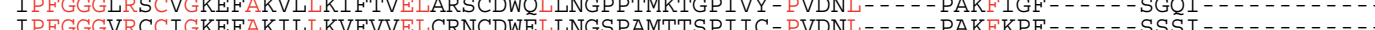
XCYP26a IPFGGGVRCIGKEFAKILLKVFVVLCRNCDWELLNGSPAMTISPIIC-PVDNL- hCYp26a 26 LPFGGGVRTCLGKHLAKLFLKVLAVELASTSRFELATRTFPRITLVPVLHPVDGL_- - SVKF- F-GLDSNONEILPETEAMLSATV mCyp26b LPFGGGVRTCLGKHLAKLFLKVLAVELASTSRFELATRTFPRITLVPVLHPVDGL-----SVKF--F-G-DSNQNEILPETEAMLSATV

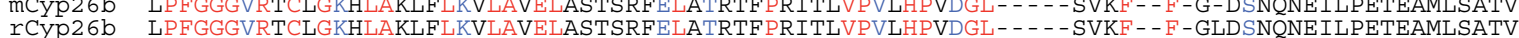
zCyp26b LPFGGGVRSCLGKOLATLFLKLTAVETAGGSRFELSTRTFPRMI SVPVVHPTDGT-- - - RVKF- - F-GLDSNONOIMAKSDEMLDATV

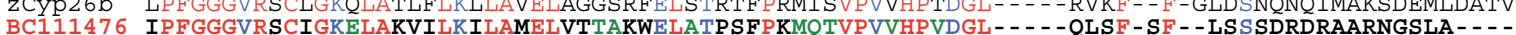

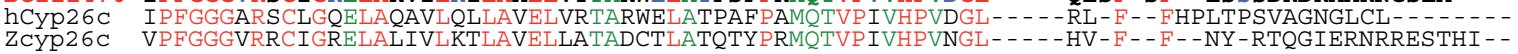

Fig. 1. Comparison of deduced amino acid sequence of BC111476 with that of several Cyp26 family genes. Amino acid residues in red are identical among all Cyp26 genes, whereas those in green are identical among only Cyp26c genes. 
Cyp26 is a RA-metabolizing factor first identified as a novel cytochrome P450 (P450RAl; White et al., 1996). Like RalDH2, Cyp26 is expressed in many species (White et al., 1997; Fujii et al., 1997). Cyp26 genes comprise at least 3 subgroups: Cyp26a, Cyp26b, and Cyp26c. Xenopus Cyp26a is found in the circumblastoporal ring and dorsal animal hemisphere, and overexpression of xCyp26a mRNA induces anteriorization (Hollemann et al., 1998; de Roos et al., 1999). Similar expression characteristics were shown in zebrafish (Kudoh et al., 2002). In fact, a recent triple knockdown of Cyp26a, Cyp26b, and Cyp26c in zebrafish caused complete loss of krox20 expression (Hernandez et al., 2007), suggesting that these three genes function coordinately in hindbrain development.

FGF and Wnt signaling both regulate $x$ Cyp26a expression (Kudoh et al., 2002). As described above, Wnt signaling is also important in AP neural patterning. Injection of a truncated form of bone morphogenetic protein receptor tBR into the ventral side of embryos induced secondary axis formation without head structure (Glinka et al., 1997). Coinjection of tBR and dominant-negative $x W n t 8$ (dn-xWnt8) induced complete axis with head structure, suggesting that inhibition of Wnt signaling is crucial for head formation (Glinka etal., 1997). Moreover, several canonical Wnt signaling (late canonical Wnt signaling) pathway components such as $\beta$-catenin and glycogen synthase kinase $-3 \beta$ (GSK-3 $\beta$ ) are also critical for head formation (Onai et al., 2004, Yamamoto et al., 2005, Michiue et al., 2004; Funato et al., 2006). However, the target genes of late canonical Wnt signaling remain unknown.

We therefore sought to identify genes expressed in the presumptive head region of early neurula and negatively regulated by late canonical Wnt signaling. To do this, we prepared cDNAs from different parts of the presumptive anterior neuroectoderm and compared gene expressions by microarray analysis; specifically, between the anterior region of early neurula from normal embryos and the anterior region of early neurula from $\beta$-catenin-injected embryos (described on M\&M section in detail). We isolated several genes that are negatively regulated by Wnt signaling. We started to characterize the molecular features of xCyp26c from this list. Different from xCyp26a, $x$ Cyp26c expression was restricted to the anterior region where it was found in some sets of cranial nerves. XCyp26c expression was positively regulated by inhibition of canonical Wnt signaling, and high-dose RA treatment induced xCyp26c expression only in the anterior region in neurula. Injection of $x$ Cyp26c mRNA into the animal pole region of Xenopus embryos rescued this posteriorizing effect of RA. Together, these results suggested an important role for xCyp26c in anterior neural development via regulating $\mathrm{RA}$ concentration in the anterior neural region.

\section{Results}

\section{Identification of XCyp26c as a head-specific gene}

We earlier identified several genes that were downregulated by activation of $\beta$-catenin in the anterior neuroectoderm of early-neurula embryo. One of these (Genbank
No. BG264135) was not described as an identified gene, but BLAST searching indicated homology with the gene for hypothetical protein MGC131069 (Genbank No. BC111476, Unigene No. $\mathrm{xl} .82105)$. The BC111476 sequence encodes a 603-amino acid protein. Homology search analysis also revealed a BC111476 gene product with an obvious Cyp (Cytochrome P450) domain (Fig. 1). Different from other Cyp26 genes, the BC111476 gene product contains a long sequence gap of about 90 amino acids in length within the Cyp26 domain (Fig. 1). Thus, we divided the $\mathrm{BC} 111476$ sequence into $\mathrm{N}$-terminal region (aa 1-342) and $\mathrm{C}$ terminal region (aa 438-602), and compared these regions among other Cyp26 gene products. The BC111476 N-terminal region was most similar with human Cyp26c (hCyp26c) (65.0\% homol-
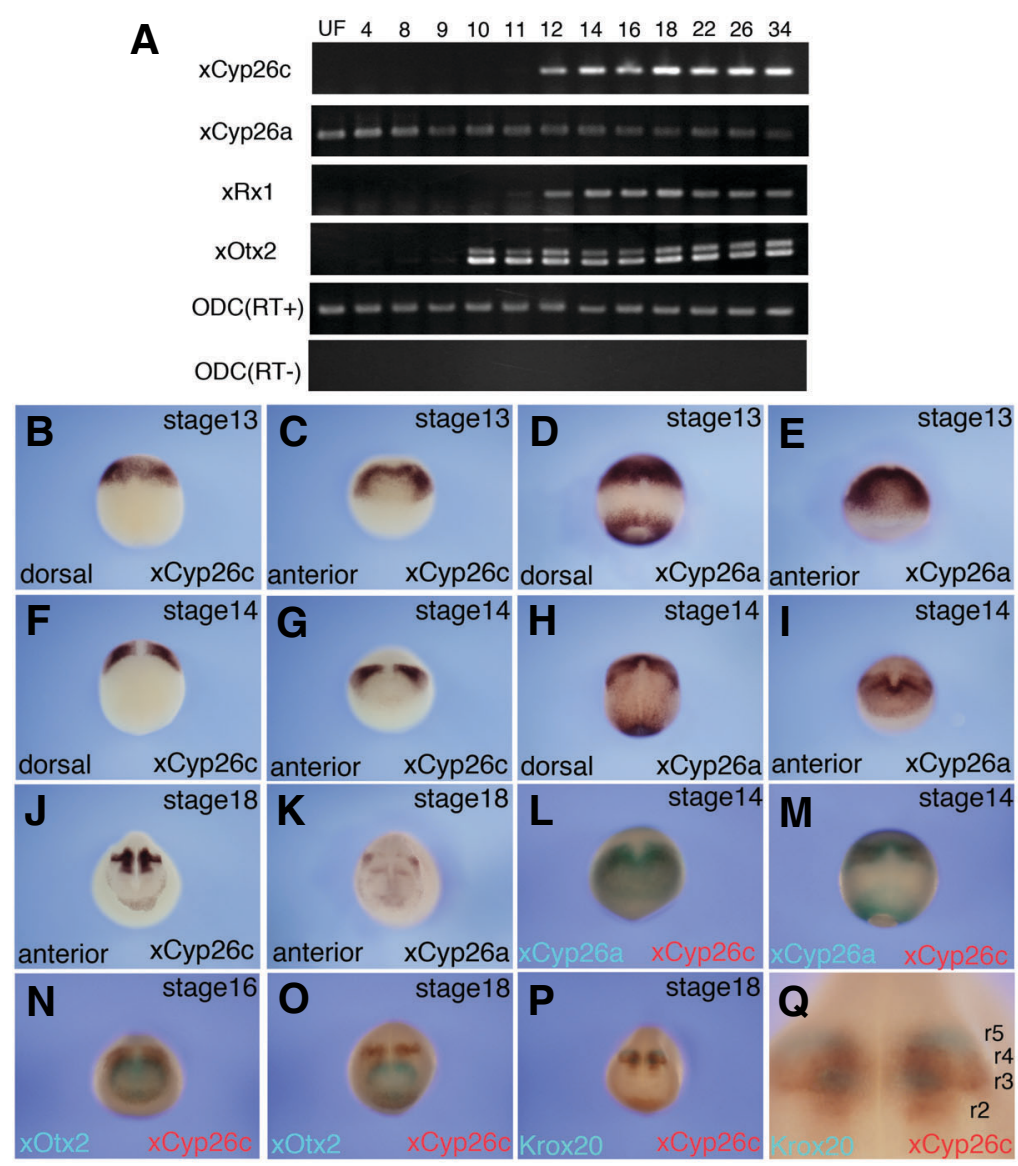

Fig. 2. Temporal and spatial pattern of $\boldsymbol{x}$ Cyp26c expression. (A) Temporal

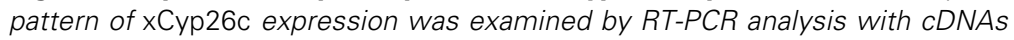
prepared from embryos at various stages. XCyp26c expression increased from the mid-late gastrula stage. This pattern is different from $\times$ Cyp26a, which is ubiquitously expressed. (B-K) The spatial pattern of xCyp26c (B, C,F,G,J) and xCyp26a $(\mathbf{D}, \mathbf{E}, \mathbf{H}, \mathbf{I}, \mathbf{K})$ expression was analyzed by whole-mount in situ hybridization. Different from $\mathrm{xCyp26a}$, $\mathrm{x}$ Cyp26c was expressed only in the presumptive anterior neural region at the early neurula stage $(B, C$, compared with $D, E)$. At the midneurula stage, the midline of $\mathrm{x} C y \mathrm{p} 26 \mathrm{c}$ expression decreased $(\mathbf{F}, \mathbf{G})$. By the late

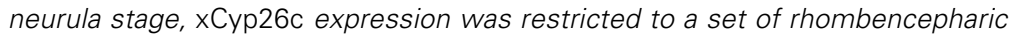
cells $\mathbf{( J )}$, whereas $x$ Cyp26a expression was greatly reduced (K). (L-Q) Double in situ hybridization. Brown staining indicates $\times$ Cyp26c expression, while the light blue staining indicates $\times$ Cyp26a (L,M), $\times 0 t \times 2(\mathbf{N}, \mathbf{0})$, and $\times k r o \times 20(\mathbf{P}, \mathbf{Q})$ staining, respectively. Anterior view $(L, N, O, P)$. Dorsal view (M). (Q) Magnified image of (M). r2-5 show rhombomeres 2 to 5, respectively. These data indicated that xCyp26cpositive cells were mainly identical with $r 2, r 3$, and $r 4$. 
ogy; Table 1), and also showed high homology with zebrafish Cyp26c (zCyp26c) (58.9\%; Table 1). Compared with Cyp26c, Cyp26a and Cyp26b showed less homology with BC111476. Similarly, the Cterminal domain of $\mathrm{BC} 111476$ was most similar to human Cyp26c (69.9\%). Therefore, we defined BC111476 as xCyp26c and further investigated the role of this gene.

\section{Expression of $\mathrm{x}$ Cyp26c gene in gastrula-neurula- stage embryo}

We next used RT-PCR to analyze the temporal expression of $x$ Cyp26c, using cDNAs prepared from various Xenopus embryonic stages. XCyp26c expression was low prior to gastrulation, and increased from the mid-gastrula stage (Fig. 2A). This was different from that of xCyp26a, which was expressed throughout development (Fig. 2A). It should be pointed out that xCyp26a expression gradually decreased after the tailbud stage (Fig. 2A). Microarray analysis revealed 40-fold higher signal intensity for $\mathrm{xCyp26c}$ expression in the anterior region compared to that in the posterior region (Table 2), suggesting that $\mathrm{xCyp26c}$ expression was restricted to the head region of neurula. To evaluate this result and further examine the spatial expression of $x$ Cyp26c, we carried out in situhybridization, using $x$ Cyp26a expres-
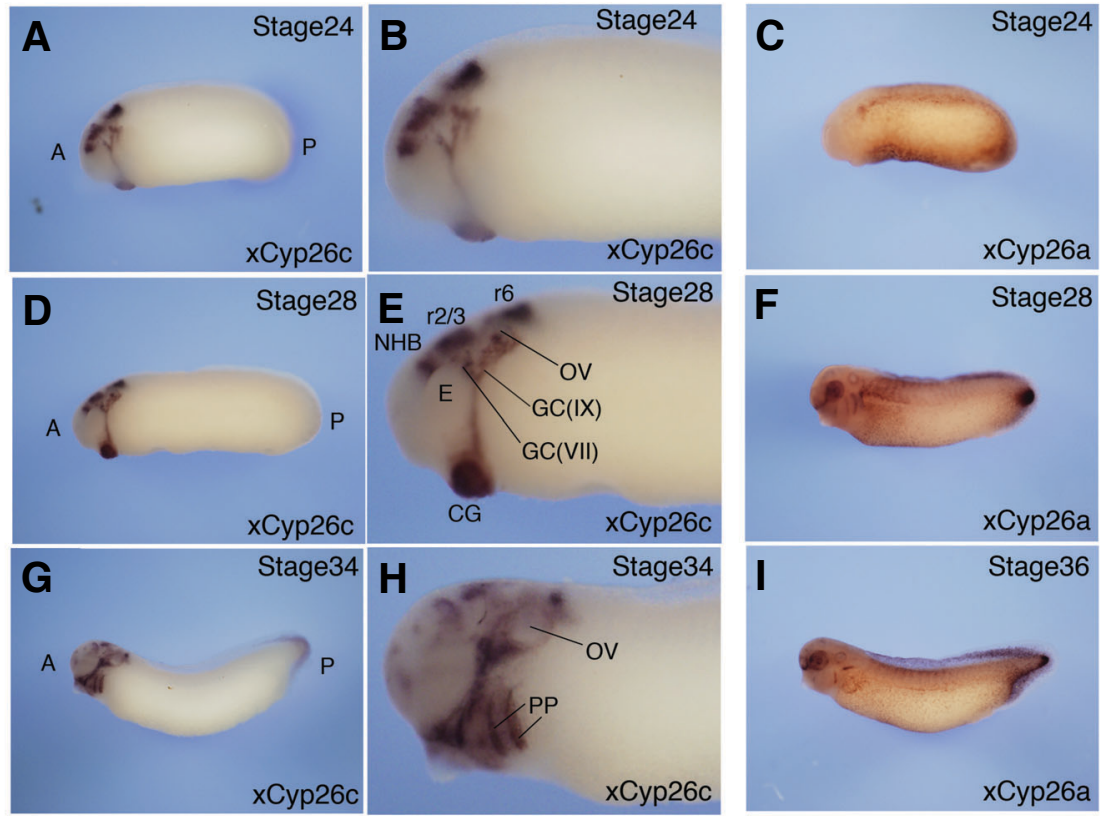

Fig. 3. Spatial expression of $\boldsymbol{x}$ Cyp26c after tailbud stage. (A-B, D-E, G-H) XCyp26c expression. (C,F,I) XCyp26a expresion. Stage 24 (A-C), stage 28 (D-F), and stage 36 (GI). XCyp26c was expressed in restricted sets of cranial nerves (GC(VII) and GC(IX)). XCyp26c was also expressed in the pharyngeal pouch (PP) and rhombomeric cells ( $22 / 3$ and $r 6)$. These patterns were obviously different from those of xCyp26a $(C, F, I)$. sion to compare spatial patterns. At stage 13, xCyp26c was expressed in presumptive anterior neuroectoderm, in an arch-like pattern similar to xCyp26a (Fig. 2C, E). In contrast to xCyp26a, however, there was no posterior expression of $x$ Cyp26c around the yolk plug (Fig. 2B, D). At stage 14, xCyp26c expression was strong in the presumptive hindbrain region and decreased around the midline (Fig. 2F, G). The expression patterns of $x$ Cyp26a and xCyp26c were similar in the anterior neuroectoderm (Fig. 2G, I); $\mathrm{xCyp26a}$ expression was also seen at the rhombencephalic region, but the expression was most intense around the midline and the anterior end of hindbrain, whereas the expression area of

TABLE 1

\section{\% HOMOLOGY BETWEEN BC111476 AND OTHER CYP26 PROTEINS}

\begin{tabular}{lcc} 
& \multicolumn{2}{c}{ \% homology } \\
\cline { 2 - 3 } & $\begin{array}{c}\text { BC111476-N } \\
\text { (a.a. 1-342 }\end{array}$ & $\begin{array}{c}\text { BC111476-C } \\
\text { (a.a. 438-602) }\end{array}$ \\
\hline hCyp26a & 45.4 & 52.0 \\
mCyp26a & 44.8 & 51.3 \\
rCyp26a & 45.1 & 52.7 \\
cCyp26a & 45.0 & 52.4 \\
xCyp26a & 45.0 & 50.3 \\
zCyp26a & 42.4 & 52.3 \\
& & 58.3 \\
hCyp26b & 54.6 & 59.5 \\
mCyp26b & 54.0 & 58.9 \\
rCyp26b & 53.7 & 60.1 \\
zCyp26b & 52.3 & 69.9 \\
hCур26c & & 65.8 \\
zCyp26c & 65.0 & \\
\hline
\end{tabular}

These data indicate that BC111476 is likely to be a Xenopus homolog of Cyp26c
xCyp26c was more posteriorly located (Fig. 2G, I). XCyp26a was moderately expressed at the anterior neural plate and adjacent regions including the presumptive cement gland (Fig. 2l). Moreover, xCyp26c was not expressed in the posterior ectoderm even at the mid-neurula stage (Fig. 2F). At stage 18, the region of $x$ Cyp26c expression was further narrowed, and one of the rhombencephalic expression areas extended laterally (Fig. 2J). Expression of xCyp26c was also seen outside of the neural plate including presumptive cement gland region at this stage (Fig. 2J). XCyp26a expression was markedly decreased at the anterior neural plate by stage 18 (Fig. 2K), whereas posterior expression remained (data not shown).

We next used double in situ hybridization with digoxygenin (DIG)-labeled and fluorescein (Flu)-labeled probes to determine the exact expression region of $x$ Cyp26c. First, we used DIGlabeled xCyp26a and Flu-labeled xCyp26c mRNAs. At the early neurula stage, expression patterns around the yolk plug were different between the two transcripts (Fig. 2M), while the intense expression patterns in the anterior neural plate seemed nearly identical, with slightly weaker expression of xCyp26c than xCyp26a at the midline (Fig. 2L). The circular patterns of expression in anterior neuroectoderm were also similar, although not identical in this region (Fig. 2M). Next, we compared the anterior and posterior regions of $x$ Cyp26c expression using a mid-brain marker gene (Xotx2). The posterior ends of the Xotx2 expression area only partially overlapped with xCyp26c expression, suggesting that $x$ Cyp26c was mainly expressed in rhombencephalon (Fig. $2 \mathrm{~N})$. At stage 18, the most anterior part of the $x$ Cyp26c expression area overlapped closely with that of Xotx2, indicating that the anterior end of $\mathrm{xCyp26c}$ expression extended into the cement gland region (Fig. 2O). To identify which rhombomeric region expressed xCyp26c, we performed double in situ hybridization 
TABLE 2

MICROARRAY SIGNAL INTENSITIES OF XCYP26A AND XCYP26C EXPRESSION

\begin{tabular}{|c|c|c|c|c|c|c|c|}
\hline \multirow[b]{2}{*}{ No. } & \multirow[b]{2}{*}{ genename } & \multicolumn{3}{|c|}{ slide\#1 } & \multicolumn{3}{|c|}{ slide\#2 } \\
\hline & & Ant. & Pos. & $A / P$ & Ant. & Post. & $A / P$ \\
\hline XI.1946 & xСyp26c & 9850 & 232 & 42.5 & 9130 & 182 & 50.2 \\
\hline \multirow[t]{2}{*}{ XI.456 } & xСур26а & 12600 & 15500 & 0.8 & 9900 & 16200 & 0.6 \\
\hline & & \multicolumn{3}{|c|}{ slide\#3 } & \multicolumn{3}{|c|}{ slide\#4 } \\
\hline No. & genename & bcat. & No & No/bcat. & No & bcat. & No/bcat. \\
\hline XI.1946 & xСyp26c & 3770 & 8380 & 2.22 & 2910 & 7100 & 2.44 \\
\hline $\mathrm{XI} .456$ & xСyp26a & 7770 & 10000 & 1.28 & 7450 & 11100 & 1.49 \\
\hline
\end{tabular}

Ant. and Pos. shows target RNAs derived from the anterior and posterior region, respectively. Expression of xCyp26c was obviously decreased by $b$-catenin (b-cat) injection.

with xCyp26c and Xkrox20 probes. As expected, stage-18 embryos showed partially overlapping rhombencephalic expressions of xCyp26c and xkrox20 on low-magnification imaging (Fig. $2 \mathrm{P})$. Higher magnifications localized the $\mathrm{xCyp} 26 \mathrm{c}$ expression to r2, r3, and r4 (Fig. 2Q).

\section{XCyp26c expression in cranial nerves at the tailbud-tad- pole stage}

At stage 24, strong xCyp26c expression was seen in cement grand primordia (Fig. $3 A$ ) and in some rhombencephalic regions. From here, the expression of $x$ Cyp26c extended to the cement glands, although the two regions of expression did not connect (Fig. 3A, B). At stage 28, xCyp26c expression in cranial nerves from the rhombencephalon further elongated, obviously connecting with that in cement glands (Fig. 3D, E). Spatial

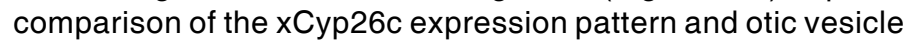
position indicated the xCyp26c-positive nerves to be typeVII and type IX (Fig. 3E). At stage 34, xCyp26c expression was still restricted in the head region (Fig. $3 G$ ). Expression in the cranial nerves was maintained and became complicated especially around the cement gland (Fig. 3H). XCyp26c expression was also apparent in the rhombencephalon was also seen, although here it was less intense and restricted (Fig. $3 \mathrm{H}$ ). Moreover, $x$ Cyp26c-positive cells were also seen at the ventral side of the otic vesicle and pharyngeal pouch (Fig. 3H). Finally, the xCyp26c expression pattern differed from that of xCyp26a (Fig. 3 C, F, I).

\section{XCyp26c expression is regulated by canonical Wnt signal- ing}

The microarray analysis suggested that canonical Wnt sig- naling regulates $x$ Cyp26c transcription, although not whether $x$ Cyp26c is a direct target gene. Thus, we next examined how modulating the Wnt pathway affected xCyp26c expression. We injected various doses of $\beta$-catenin mRNA into the animal poles of 8-cell embryos and assayed expression by RT-PCR analysis and in situ hybridization. Injection of $1 \mathrm{ng}$ of $\beta$-catenin mRNA decreased xCyp26c expression (Fig. 4A, column 1), but had little effect on the expression of xCyp26a (Fig. 4A, column 2). In situ hybridization of the $\beta$-catenin-injected embryos revealed clearly reduced $x$ Cyp26c expression in the anterior neural plate (Fig. 4D, E). These results suggested that $x$ Cyp26c expression is negatively regulated by canonical Wnt signaling.

\section{XCyp26c expression was upregulated by retinoic acid treat- ment}

Cyp26a expression changes in response to low doses of RA (1.8 x 10-7 M; Hollemann et al., 1998). Conversely, in chick embryo, Cyp26c was downregulated by RA treatment (Reijntjes et al., 2005). We used in situhybridization to assess the effect of RA treatment on xCyp26c expression. As described above, normal embryos showed intense expression of xCyp26c mRNA in the rhombencephalic region of mid-neurula stages (Fig. 5A, Fig 2G). Increasing the concentration of RA gradually shifted the $\mathrm{xCyp26c}$ expression area to the anterior end of the neuroectoderm (Fig. 5B, C, D, and E). High magnification images showed that most anterior edges of xCyp26c expression were not shifted, whereas the rhombencephalic expression was slightly extended and shifted both anteriorly and medially, resulting in overall reduction of the expression area (Fig. 5E, compared with $5 \mathrm{~A}$ ). The $\mathrm{xCyp26a}$ expression pattern was also
A

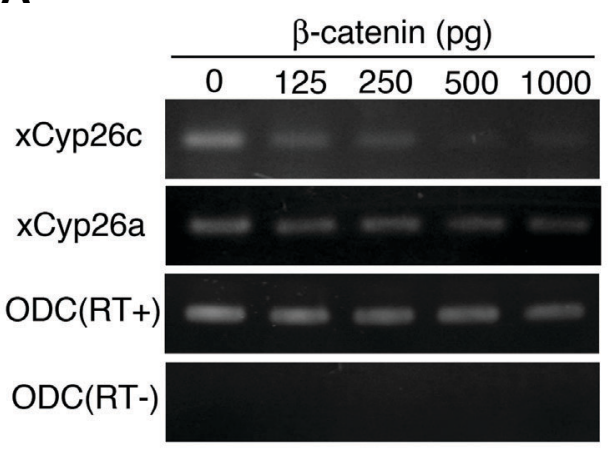

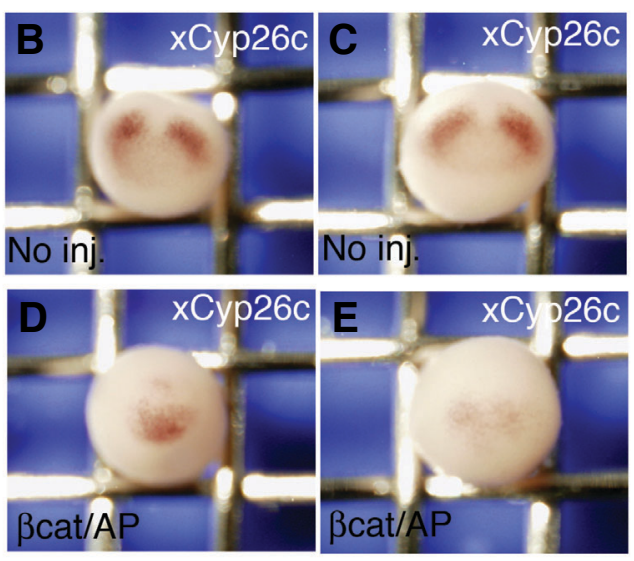

Fig. 4. Regulation of $x$ Cyp26c expression by canonical Wnt signaling. (A) RT-PCR analysis with cDNAs synthesized using $\beta$-catenin-injected embryos. Injection of $\beta$-catenin mRNA into the animal pole region of embryos decreased xCyp26c expression in a dose-dependent manner. XCyp26a expression was not clearly decreased by $\beta$-catenin injection. (B-E) Expression of xCyp26c in mid-neurula embryos injected into the animal pole with nothing $\mathbf{( B , C )}$ or $\beta$-catenin (D,E). $\beta$-catenin clearly inhibited $\times$ Cyp26c expression $(D, E)$. 

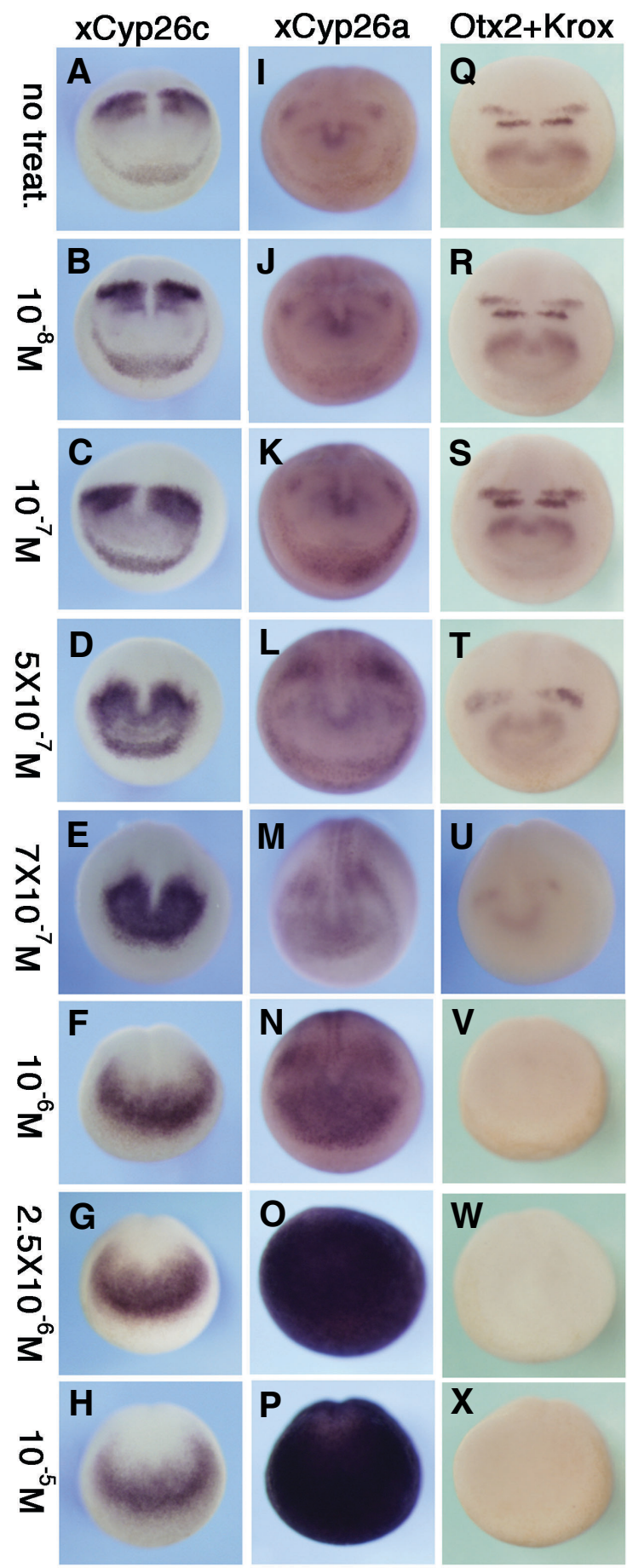

Y

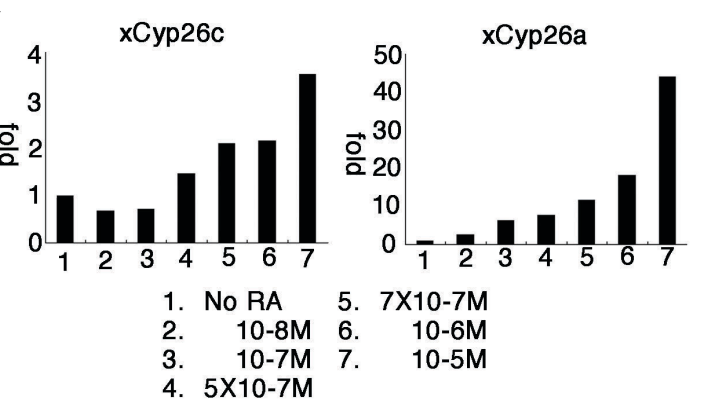

changed by RA treatment, although not in the same manner as

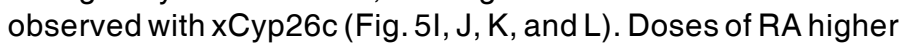
than $10^{-6} \mathrm{M}$ no longer affected the $\mathrm{xCyp26c}$ expression pattern, which remained condensed in an arched shape (Fig. $5 \mathrm{~F}, \mathrm{G}$, and $H)$. In contrast, xCyp26a became expressed throughout the whole embryo as the RA dose increased above $10^{-6} \mathrm{M}$ (Fig. $5 \mathrm{~N}$, $\mathrm{O}$ and $\mathrm{P}$ ). We also observed $\mathrm{xOtx} 2$ and $\mathrm{xkrox} 20$ gene expressions in these embryos. Up to $7 \times 10^{-7} \mathrm{M} \mathrm{RA}$, the marker gene expression patterns shifted similarly to that of $x$ Cyp26c, before disappearing with RA concentrations above $10^{-6} \mathrm{M}$ (Fig. 5Q-V). Furthermore, we ensured change of the expression level of xCyp26c and xCyp26a by real-time PCR analysis. XCyp26c

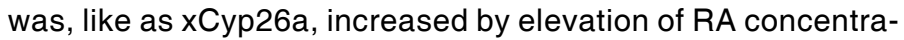
tion (Fig. 5Y). Though expression pattern did not show obvious difference, $\mathrm{xCyp26c}$ expression was further increased more than $10^{-6} \mathrm{M}$ RA (Fig. $5 \mathrm{Y}$ lane 6 and 7, compared with Fig. 5F and H).

\section{Overexpression of XCyp26c could attenuate the anterior neural RA effect}

To examine whether overexpression of $x$ Cyp26c induces morphogenesis of the anterior neural region, we injected $x$ Cyp26c mRNA into Xenopus embryos and observed the morphological effects. Injection of $1 \mathrm{ng}$ of $x$ Cyp26c mRNA into the dorsal blastomere occasionally induced a small head, although the phenotype was weak (Fig. 6D). However, histological examination of the injected embryos revealed no morphological change in brain structure (Fig. 6E, F, compared with B and C). Ventral injection with $\mathrm{xCyp26c}$ caused no defect (data not shown).

Next we speculated that if $x$ Cyp26c regulates patterning in the anterior neural region at the neurula stage, overexpression of $x$ Cyp26c could weaken the observed RA effect. Accordingly, we treated the $x$ Cyp26c mRNA-injected embryos with RA and observed the external morphology, while mock-injected embryos treated with $10^{-6} \mathrm{M}$ RA showed loss of head structure due to posteriorization (Fig. 6G). On the other hand, $1 \mathrm{ng}$ of $\mathrm{xCyp} 26 \mathrm{c}$ mRNA injected into animal poles still caused head defects, but the severity was less, and tadpoles still showed eye structures (Fig. 6H). Subsequent RT-PCR experiments on lacZ mRNAinjected embryo treated with $10^{-6} \mathrm{M}$ RA showed decreased expression of both $x B F-1$ (telencephalic marker) and $x R \times 1$ (diencephalic marker) (Fig. 6J, lanes 2 and 3). Conversely, the decrease was significantly attenuated in xCyp26c-injected embryos (Fig. 6J, lanes 5 and 6). These results suggested that $x$ Cyp26c expression rescues the loss of forebrain marker ex-

Fig. 5. Effect of retinoic acid (RA) on $x$ Cyp26c expression. (A-X) Embryos were treated with various doses of RA at stage 8, cultured until stage 13, and then prepared for WISH analysis. No treatment (A-C). RA treatment (D-X). Concentration of $R A(M)$ is shown on left side. Expression pattern of xCyp26c (A-E), xCyp26a (I-P) and xOtx2 and xkrox20 (Q$\mathbf{X}$. Moderate doses of RA increased $\times$ Cyp26c expression, but narrowed the area of expression (A-M). Over $10^{-6} \mathrm{M}$ of $R A, \mathrm{xCyp} 26 \mathrm{c}$ expression became restricted in the anterior neuroectoderm and underwent no further change (F-H). XCyp26a was expressed throughout the embryo (O,P), whereas xOtx2 expression completely disappeared (V-X). (Y) Real-time PCR analysis with whole embryos treated with RA. These graphs are expressed as ratio of expression level of RA-treated embryo (lane 2-7) to that of no-RA treated embryo (lane 1). 

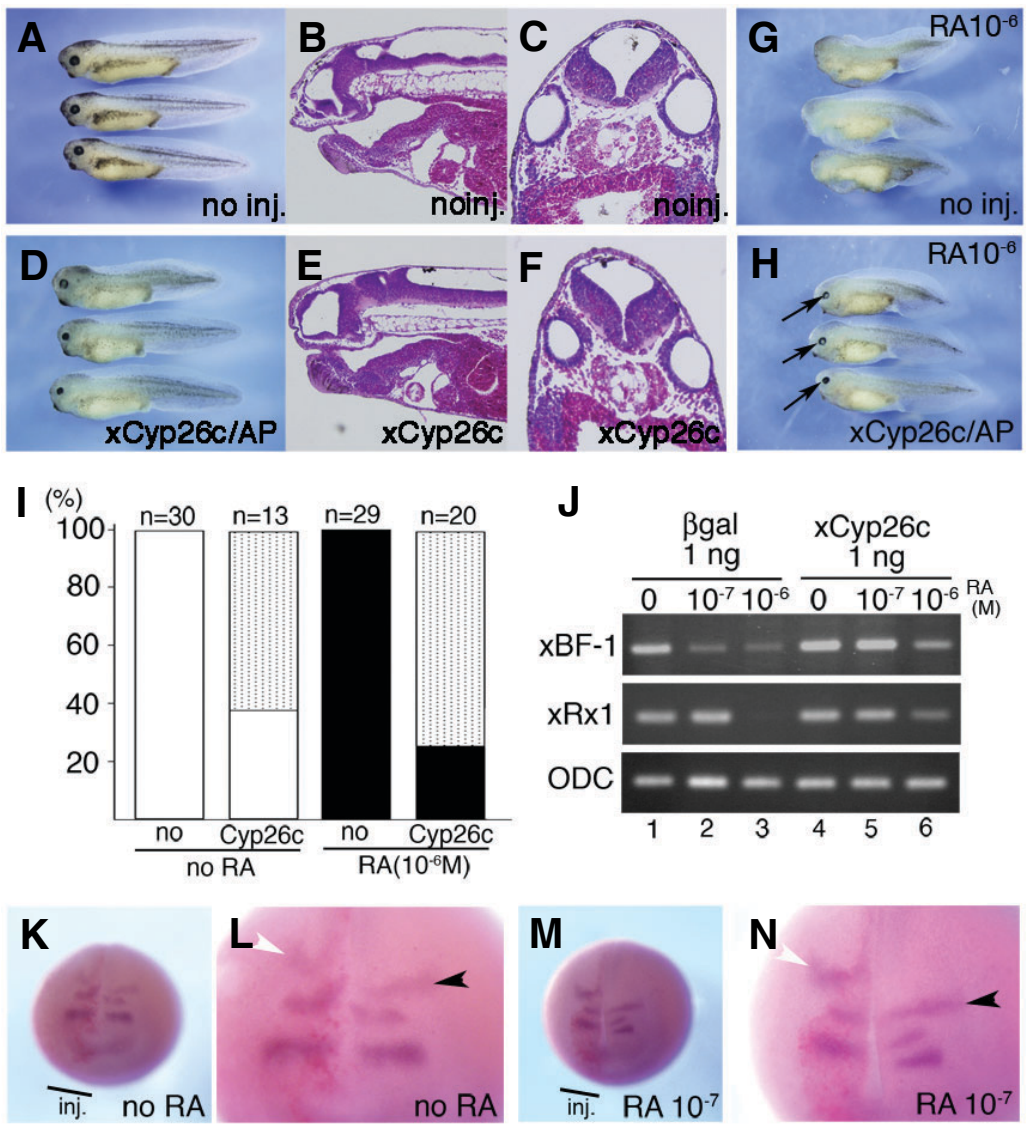

Fig. 6. Overexpression of $x$ Cyp26c could attenuate retinoic acid (RA)-induced posteriorization. (A-F) Phenotype of tadpole injected with $\times$ Cyp26c mRNA. (A-C) Normal tadpoles. (DF) Tadpoles injected with $1 \mathrm{ng}$ of $\mathrm{x}$ Cyp26c $\mathrm{mRNA}$ into the animal pole region. (B,C,E,F) Histological section of $\mathrm{XCyp} 26 \mathrm{c}$-injected tadpoles. Sections were stained by hematoxylin-eosin. (B,E) Sagittal sections, whereas $(C, F)$ show transverse sections of $(A, D)$, respectively. (G-I) Head defect by $R A$ treatment was rescued by $\times C y p 26 c$ injection. Embryos not injected $(G)$ or

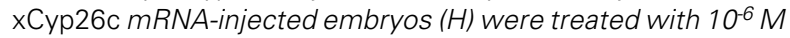
of RA. In xCyp26c-injected embryos, loss of eye structure was rescued (arrow). These results are summarized in graph (I). White box, dotted box, and filled box show normal eye, small eye, and no eye structure, respectively. Point out that the size of eye vesicle reflects the severity of posteriorizaion by $R A$ treatment. (J) RT-PCR analysis with cDNAs synthesized from whole embryo injected with $\beta$-gal (1 ng; lanes 1-3) or xCyp26c (1 ng; lanes 4-6). Embryos were treated with $10^{-7} \mathrm{M}$ (lane 2, 5) or $10^{-6} \mathrm{M}($ lane 3,6$)$ of $R A$, or untreated (lane 1, 4). Overexpres-

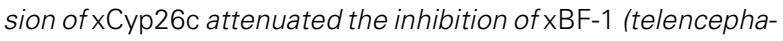
lic marker; column 1) and XRX1 (diencephalic marker; column 2) expression by RA treatment (lane 6). (K-N) Change of expression patterns of En2 and xkrox20 by xCyp26c injection. $(L, N)$ are magnified views of $(K, M)$, respectively. Injected side (inj. In (K) and (M)) was indicated by lineage tracer (Red-gal). (K,L) show no RA-treated embryos and $(\mathbf{M}, \mathbf{N})$ show $10^{-7} \mathrm{M}$ RA-treated embryos. Normal embryos showed a slight posterior shift of xkrox20 expression in the xCyp26c-injected area (white arrowhead, compared with noninjected region (black arrowhead)). RA-treated embryos showed an obvious difference in xkrox 20 expression patterns between injected and noninjected regions. pression induced by $\mathrm{RA}$ treatment. To observe this rescue spatially, we injected xCyp26c mRNA into half the blastomere of animal poles and treated with RA. Analysis of anterior marker-gene expressions by in situ hybridization showed the expected anterior shift in En2 and xkrox20 expression in xCyp26c mRNA-injected embryos (Fig. $6 \mathrm{~K}, \mathrm{~L}$ ), and treatment with 10-7 $M$ RA increased this shift (Fig. $6 \mathrm{M}, \mathrm{N}$ ).

\section{Discussion}

\section{XCyp26c expression in anterior ectoderm}

In situ hybridization experiments showed xCyp26c expression restricted to the anterior region of early neurula, while xCyp26a was expressed both in anterior neuroectoderm and around the yolk plug (Hollemann et al., 1998). In support of this, our microarray analysis indicated 40-fold higher expression of $x$ Cyp26c in the anterior embryonic regions than in posterior parts, whereas abundant $x$ Cyp26a expression was seen posteriorly (Table 2). RA has a posteriorizing effect and this phenotype is severe only in the head region. We therefore propose that $x$ Cyp26c functions mainly in AP axis formation.

After the late neurula stage, xCyp26c was strongly expressed in a rhombencephalic region that corresponded to $r 2$, $\mathrm{r} 3$, and $\mathrm{r} 4$ by double in situhybridization. These data correlate with a recent zebrafish study (Hernandez et al., 2007), showing evolutionary conservation. At the tadpole stage, xCyp26c was expressed only in a subset of cranial nerves. Neuroanatomically, the root of cranial nerve VII is $r 4$, whereas nerve IX is r6, supporting the rhombencephalic expression of xCyp26c. Fur- thermore, our results showed these nerves extending to cement gland primordial, and nerves VII and IX are related to saliva secretion, supporting this result.

\section{The effect of RA on xCyp26c expression}

In this study, xOtx2 expression shifted anteriorly, indicating shrinkage of the forebrain region. XCyp26c expression showed a similar shift until the RA concentrations exceeded $10^{-6} \mathrm{M}$, when expression did not change further temporally or spatially. At these RA levels, xkrox20 and En2 expression completely disappeared. These results may show existence of two independent systems for regulating $\mathrm{xCyp26c}$ transcription. XCyp26c expression was not quantitatively enhanced at low RA levels, with the xCyp26c expression area dependent simply on AP patterning. On the other hand, exposure to RA in high concentrations expanded xCyp26c expression only in the limited region of embryos. In addition and unlike for other family member xCyp26a, the area of ectopic $x$ Cyp26c expression was limited. This limitation might reflect that

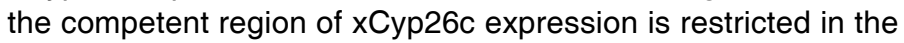
anterior neuroectoderm. It is unclear, however, how this competent area is defined and further studies need to be done.

\section{XCyp26c function in rhombencephalon}

Our results revealed strong xCyp26c expression in the rhombencephalic region. RA signaling is involved with rhombencephalic differentiation (Papaopulu et al., 1991; Kolm et al., 1997), so we predicted that overexpression of XCyp26c would cause defects in hindbrain development. However, xCyp26c injection had no obvious effect on rhombencephalon, suggesting 
that RA signaling in hindbrain is sufficiently maintained at low RA levels and that any effect of $x$ Cyp26c is hard to observe. We have not yet done downregulation assays with morpholino antisense oligos, and recent studies in mouse and zebrafish showed that knockdown of only xCyp26c had almost no effect, and that simultaneous disruption of both $\mathrm{xCyp26a}$ and $\mathrm{xCyp26c}$ was required for the hindbrain defect (Uehara et al., 2007; Hernandez et al., 2007). Together with the expression patterns of these genes, these findings implicate a cooperative action of xCyp26a and xCyp26b, in addition to xCyp26c alone, in rhombencephalon development.

\section{Transcriptional regulation of RA signaling-related genes by Wnt signaling}

Finally, transcription of xCyp26c was inhibited by upregulation of canonical Wnt signaling. Interestingly, our microarray analysis revealed that RaIDH2, a RA synthesizing-factor gene, was positively regulated by canonical Wnt signaling (data not shown). It is thought that a presumptive gradient of canonical Wnt signaling is formed along with the AP axis. We therefore propose that formation of a RA gradient that is high in the posterior region and low in the anterior region might be actualized by complementary expression of xCyp26c and RaIDH2 via Wnt signaling. However, xCyp26a is expressed both anteriorly and posteriorly, suggesting that RA signal regulation for AP pattern determination is complicated. More analyses are clearly needed to understand the crosstalk between RA and Wnt signaling in AP neural patterning.

\section{Materials and Methods}

\section{DNA construction}

A DNA fragment containing the xCyp26c gene was obtained by RTPCR (described below). Primers for cloning were 5'-AGG ACT GTC ACT AGT GAG ACC ACT GG-3' and 5'-TCA GGC AAG TGA CCC ATT TCT TGC TGC-3'. For microinjection, the xCyp26c fragment was inserted into the EcoRI and Xhol sites of pCS2+.

\section{RT-PCR}

RT-PCR analysis was performed as described above (Suzawa et al., 2007). Primers for PCR were as follows: xCyp26c; 5'-ACG AGG GGA AAC TGG GCA AAT TCA AC-3' and 5'-TCA GGC AAG TGA CCC ATT TCT TGC TGC-3', xCyp26a; 5'-CTT GCG GAG GTG GAG TGA GGT G3' and 5'-GCT TAA ATA GAG CTG GAG AAG GG-3', ODC; 5'-GCC ATT GTG AAG ACT CTC TCC ATT C-3' and 5'-TTC GGG TGA TTC CTT GCC AC-3', xRX1; 5'-GGC TAT GGA GAT CCA TAT TCA GG-3' and 5'-CTC TTC TCT GCT GTA TAC GTC GG-3', xOtx2; 5'-GGA TGG ATT TGT TAC ATC CGT C-3' and 5'-CAC TCT CCG AGC TCA CTT CCC-3'.

Real time PCR analysis was performed with Chromo4 real-timePCR analysis system (BioRad). For detection, we used iQ CYBR Green Supermix (BioRad).

\section{Microinjection}

Messenger RNAs for microinjection were synthesized with a mMessage mMachine SP6 kit (Ambion) and were cleaned up with a RNeasy RNA purification kit (QIAGEN). Embryos were obtained by artificial fertilization, dejellied with $4.6 \%$ L-cysteine solution, and then microinjected using a PLI100 microinjector (Harvard Medical Instruments). For phenotype observation, injected embryos were cultivated in $0.1 \times$ Steinberg's solution for 3 days.

\section{In situ hybridization (WISH)}

WISH was performed as described in Michiue et al. (2007). In brief, embryos were fixed in MEMFA solution (formaldehyde-MOPS solution), bleached in hydrogen peroxide-methanol, and then dehydrated using ethanol solutions. After rehydration, embryos were hybridized with DIGlabeled probe for 24 hours at $60^{\circ} \mathrm{C}$. Embryos were then incubated with $2000 x$ anti-DIG antibody (Roche) for $12 \mathrm{~h}$, washed 5 times, and detected by reaction in NBT/BCIP solution. Embryos injected with $\beta$-gal mRNA to mark the injection area were stained with Red-Gal before MEMFA fixation. For double-labeled WISH, RNA probes were labeled with DIG or Flu RNA mixture (Roche) and detected with BCIP or Vectorblack AP substrates (VECTOR) respectively.

\section{Microarray analysis}

Overview of the microarray analysis we performed was described previously (Michiue et al., 2007). For synthesizing target RNAs, stage 13 embryos were cut into three equal parts (anterior, middle, posterior) and total RNA was prepared from the anterior part and the posterior part of dissected embryo.

\section{Acknowledgment}

This work was supported in part by a Grant-in-Aid for Scientific Research from the Ministry of Education, Culture, Sports, Science and Technology of Japan (M. A. and T. M) and a Grant from the National Institute of Advanced Industrial Science and Technology.

\section{References}

ARIIZUMI T, ASASHIMA M. (2001). In vitro induction systems for analyses of amphibian organogenesis and body patterning. Int J Dev Biol. 45:273-279.

CHEN Y, POLLET N, NIEHRS C, PIELER T. (2001). Increased XRALDH2 activity has a posteriorizing effect on the central nervous system of Xenopus embryos. Mech Dev. 101, 91-103.

FUJII H, SATO T, KANEKO S, GOTOH O, FUJII-KURIYAMA Y, OSAWA K, KATO S, HAMADA H. (1997). Metabolic inactivation of retinoic acid by a novel P450 differentially expressed in developing mouse embryos. EMBO J. 16, 4163-73.

FUNATO Y, MICHIUE T, ASASHIMA M, MIKI H. (2006). The thioredoxin-related redox-regulating protein nucleoredoxin inhibits Wnt-beta-catenin signalling through dishevelled. Nat Cel/ Biol. 8, 501-508.

GLINKA A, WU W, ONICHTCHOUK D, BLUMENSTOCK C, NIEHRS C. (1997). Head induction by simultaneous repression of $\mathrm{Bmp}$ and Wnt signalling in Xenopus. Nature. 389, 517-9.

HERNANDEZ, RE, PUTZKE1, AP, MYERS, JP, MARGARETHA, L AND MOENS, CB. (2007) Cyp26 enzymes generate the retinoic acid response pattern necessary for hindbrain development. Development. 134, 177-187.

HOLLEMANN, T. CHEN, Y, GRUNZ, H AND PIELER, T. (1998) Regionalized metabolic activity establishes boundaries of retinoic acid signalling. EMBO J. 17, 7361-7372

HSU LC, CHANG WC, YOSHIDA A. (2000) Mouse type-2 retinaldehyde dehydrogenase (RALDH2): genomic organization, tissue-dependent expression, chromosome assignment and comparison to other types. Biochim Biophys Acta. 1492, 289-293.

KOLM PJ, APEKIN V, SIVE H. (1997) Xenopus hindbrain patterning requires retinoid signaling. Dev Biol. 192:1-16.

KUDOH T, WILSON SW, DAWID IB. (2002) Distinct roles for Fgf, Wnt and retinoic acid in posteriorizing the neural ectoderm. Development 129, 4335-4346.

MICHIUE T, DANNO, H., TANIBE, M., IKUZAWA, M., ASASHIMA, M. (2007). Xenopus galectin-Vla shows highly specific expression in cement glands and is regulated by canonical Wnt signaling. Gene. Expr. Patterns. 7, 852-857.

MICHIUE T, FUKUI A, YUKITA A, SAKURAI K, DANNO H, KIKUCHI A, ASASHIMA M. (2004). XIdax, an inhibitor of the canonical Wnt pathway, is required for anterior neural structure formation in Xenopus. Dev Dyn. 230, 79-90.

NIEHRS C. (1999). Head in the WNT: the molecular nature of Spemann's head organizer. Trends Genet. 15, 314-319.

ONAI T, SASAI N, MATSUI M, SASAI Y. (2004). Xenopus XsalF: anterior neuroectodermal specification by attenuating cellular responsiveness to $\mathrm{Wnt}$ signaling. Dev Cell. 7, 95-106. 
PAPALOPULU N, CLARKE JD, BRADLEY L, WILKINSON D, KRUMLAUF R, HOLDER N. (1991). Retinoic acid causes abnormal development and segmental patterning of the anterior hindbrain in Xenopus embryos. Development. 113, 1145-1158.

REIJNTJES S, BLENTIC A, GALE E, MADEN M. (2005) The control of morphogen signaling: Regulation of the synthesis and catabolism of retinoic acid in the developing embryo. Dev. Biol. 285, 224-237.

DE ROOS K, SONNEVELD E, COMPAAN B, TEN BERGE D, DURSTON AJ, VAN DER SAAG PT. (1999). Expression of retinoic acid 4-hydroxylase (CYP26) during mouse and Xenopus laevis embryogenesis. Mech Dev. 82, 205-211.

RUIZ I ALTABA A, JESSELL T. (1991). Retinoic acid modifies mesodermal patterning in early Xenopus embryos. Genes Dev. 5:175-87.

SASAIY, DE ROBERTIS EM. (1997). Ectodermal patterning in vertebrate embryos. Dev Biol. 182: 5-20.

SUZAWA K, YUKITA A, HAYATA T, DANNO H, MICHIUE T, CHO KW, ASASHIMA M. (2007). Xenopus Glucose transporter 1 (xGLUT1) is required for gastrulation movement in Xenopus laevis. Int. J. Dev. Biol. 51:183-189.

UEHARA, M., YASHIRO, K., MAMIYA, S., NISHINO, J., CHAMBON, P., DOLLE, P. and SAKAI, Y. (2007). CYP26A1 and CYP26C1 cooperatively regulate anterior- posterior patterning of the developing brain and the production of migratory cranial neural crest cells in the mouse. Dev. Biol. 302: 399-411.

WANG X, PENZES P, NAPOLI JL. (1996). Cloning of a cDNA encoding an aldehyde dehydrogenase and its expression in Escherichia coli. Recognition of retinal as substrate and role in retinoic acid synthesis. J Biol Chem. 271, 16288-93.

WHITE JA, GUO YD, BAETZ K, BECKETT-JONES B, BONASORO J, HSU KE, DILWORTH FJ, JONES G, PETKOVICH M. (1996). Identification of the retinoic acid-inducible all-trans-retinoic acid 4-hydroxylase. J Biol Chem. 271, 2992229927.

WHITE JA, BECKETT-JONES B, GUO YD, DILWORTH FJ, BONASORO J, JONES G, PETKOVICH M. (1997). cDNA cloning of human retinoic acidmetabolizing enzyme (hP450RAl) identifies a novel family of cytochromes P450. J Biol Chem. 272, 18538-41.

YAMAMOTO A, NAGANO T, TAKEHARA S, HIBI M, AIZAWA S. (2005). Shisa promotes head formation through the inhibition of receptor protein maturation for the caudalizing factors, Wnt and FGF. Cel/120, 223-35.

ZHAO D, MCCAFFERY P, IVINS KJ, NEVE RL, HOGAN P, CHIN WW, DRÄGER UC. (1996) Molecular identification of a major retinoic-acid-synthesizing enzyme, a retinaldehyde-specific dehydrogenase. Eur J Biochem. 240: 15-22. 


\section{Further Related Reading, published previously in the Int. J. Dev. Biol.}

See our Special Issue Fertilization in honor of David Garbers and edited by P.M. Wassarman and V.D. Vacquier at: http://www.ijdb.ehu.es/web/contents.php?vol=52\&issue=5/6

See our Special Issue Evolution \& Development edited by Jaume Baguna and Jordi García-Fernández at: http://www.intjdevbiol.com/web/contents.php?vol=47\&issue=7-8

The role of XTRAP-gamma in Xenopus pronephros development Dong-hui Li, Techuan Chan, Reiko Satow, Shinji Komazaki, Kouhei Hashizume and Makoto Asashima Int. J. Dev. Biol. (2005) 49: 401-408

Systematic screening for genes specifically expressed in the anterior neuroectoderm during early Xenopus development Noriyuki Takahashi, Naoko Tochimoto, Shin-Ya Ohmori, Hiroshi Mamada, Mari Itoh, Masako Inamori, Jun Shinga, Shin-Ichi Osada and Masanori Taira

Int. J. Dev. Biol. (2005) 49: 939-951

Systematic screening for genes specifically expressed in the anterior neuroectoderm during early Xenopus development Noriyuki Takahashi, Naoko Tochimoto, Shin-Ya Ohmori, Hiroshi Mamada, Mari Itoh, Masako Inamori, Jun Shinga, Shin-Ichi Osada and Masanori Taira

Int. J. Dev. Biol. (2005) 49: 939-951

Antero-posterior patterning of the vertebrate digestive tract: 40 years after Nicole Le Douarin's PhD thesis Anne Grapin-Botton

Int. J. Dev. Biol. (2005) 49: 335-347

Isolation and growth factor inducibility of the Xenopus laevis Lmx1b gene.

Caroline E Haldin, Sarjit Nijjar, Karine Massé, Mark W Barnett and Elizabeth A Jones

Int. J. Dev. Biol. (2003) 47: 253-262

Twist functions in mouse development.

Meredith P O'Rourke and Patrick P L Tam

Int. J. Dev. Biol. (2002) 46: 401-413

Goosecoid and cerberus-like do not interact during mouse embryogenesis.

Ana C Borges, Sara Marques and José A Belo

Int. J. Dev. Biol. (2002) 46: 259-262

2006 ISI **Impact Factor $=3.577^{* *}$

Getting your head around Hex and Hesx1: forebrain formation in mouse.

J P Martinez-Barbera and R S Beddington

Int. J. Dev. Biol. (2001) 45: 327-336

The role of the homeodomain protein Bozozok in zebrafish axis formation.

L Solnica-Krezel and W Driever

Int. J. Dev. Biol. (2001) 45: 299-310

Expression of the transcription factor slug correlates with growth of the limb bud and is regulated by FGF-4 and retinoic acid.

P G Buxton, K Kostakopoulou, P Brickell, P Thorogood and P Ferretti

Int. J. Dev. Biol. (1997) 41: 559-568

Retinoic acid receptors and nuclear orphan receptors in the development of Xenopus laevis.

$\mathrm{C}$ Dreyer and $\mathrm{H}$ Ellinger-Ziegelbauer

Int. J. Dev. Biol. (1996) 40: 255-262

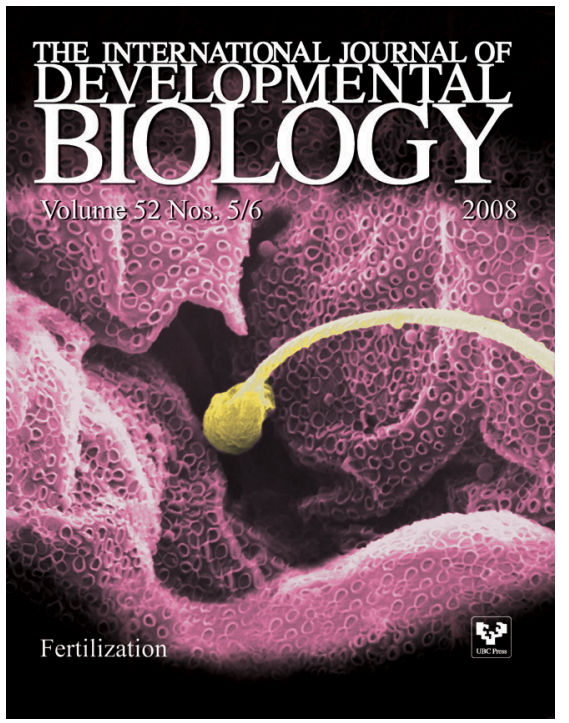

